\title{
Nuclear Factor Erythroid 2-related Factor 2 (Nrf2) as A Therapeutical Target in Type-2 Diabetes Mellitus: A Review
}

\author{
Asri Hendrawati* \\ Department of Biochemistry, Faculty of Medicine, Universitas Islam Indonesia, Jl. Kaliurang KM 14.5 Umbulmartani, Sleman, \\ Daerah Istimewa Yogyakarta, Indonesia \\ *Corresponding author. E-mail: asri_xabi@yahoo.com
}

Received date: Jan 15, 2017; Revised date: May 26, 2017; Accepted date: May 29, 2017

\section{Abstract}

$\mathrm{B}$ ACKGROUND: Increasing free radicals and oxidative stress due to chronic hyperglycemia in type-2 diabetes mellitus (DM) promotes the activity of endogenic antioxidative genes. Nuclear factor erythroid 2-related factor 2 (Nrf2) expression and activity are important to regulate the production of endogenic antioxidative enzymes.

CONTENT: Normally, Nrf2 is bound by protein Kelchlike ECH-associated protein-1 (Keap1) in the cytosol. Stimulation from oxidative stress causes the release of Nrf2 from Keap1. When activated, Nrf2 enters the nucleus and activates the antioxidant response element (ARE). This will further increase the production of antioxidative enzymes, such as catalase, nitrite oxydase and heme oxygenase- 1 . The discovery of oxidative stress, as the cause of complications in DM, gives rise to the idea of developing a treatment which can increase the expression and activity of $\mathrm{Nrf}$, one of which is a flavonoid antioxidant.

SUMMARY: Currently, nuclear factor erythroid 2-related factor 2 is an important target in the therapy of DM.

KEYWORDS: Nrf2, type-2 diabetes mellitus, endogenic antioxidative enzymes, oxidative stress, antioxidants

Indones Biomed J. 2017; 9(2): 73-7

\section{Introduction}

Diabetes mellitus (DM) is a chronic metabolic disorder which causes many complications. In 2005, the disease was reportedly affecting 135 million people. This accounts for $3 \%$ of the total world population, including poor residents in rural and urban areas.(1) The number of people in the world suffering from DM is predicted to reach 522 million in 2030.(2) According to data from the National Institutes of Health (NIH), around $90-95 \%$ cases of DM are type-2 DM.(3) In 2000, WHO reported that around 8.4 million people in Indonesia were affected by the disease. This number is projected to reach 21.3 million in 2030.(4) This large number of DM cases, as well as its constant increase, imposes a major burden on the country, especially due to the need to fund the health expenses required for treatment. Furthermore, DM also reduces the quality of life and productivity of its patients. Therefore, management of DM through using natural and affordable materials, in addition to current antidiabetic drugs, should continue to be developed.

Complications relating to DM are the leading cause of death in DM patients. Chronic hyperglycemia is the initial cause of microvascular complications, such as retinopathy, neuropathy and nephropathy, as well as macrovascular complications, especially cardiomyopathy. Hyperglycemia stimulates the production of pathological oxidants, increases peroxidation of lipid and disrupts cellular signaling pathways, such as phospholipid or kinase, increasing the oxidative stress of cells.(5) The increase of reactive oxygen species (ROS) has been known as the main cause of cellular death in DM patients. Chronic hyperglycemia promotes the production of ROS and peroxidation of lipid, increasing the oxidative stress that may damage the muscle cells.(6)

Efforts to prevent complications of DM include reducing the excessive production of ROS. This can be achieved through a scavenging mechanism by the endogenic 
antioxidants, such as superoxide dismutase (SOD). Production of endogenic antioxidants is regulated mainly by the nuclear factor erythroid 2-related factor 2 ( Nrf2) system. An activated $\mathrm{Nrf} 2$ will induce the antioxidant response element (ARE). Furthermore, the ARE will stimulate transcription of genes that code endogenic antioxidative enzymes, such as SOD, heme oxygenase (HO-1), catalase, and glutathione S-transferase (GST). Normally, Nrf2 forms a complex with Kelchlike ECH-associated protein-1 (Keap1) in the cytoplasm. Cellular injury due to oxidative stress promotes the release of Nrf2 from Keap1, then free Nrf2 will be activated and enter the nucleus to stimulate the genes producing endogenic antioxidants.(7)

Increasing Nrf2 activity in the presence of ROS, or the administration of antioxidants, has been widely proven to reduce oxidative stress. Previous studies shows that Nrf2 plays an important role in the in vitro protection of cardiac muscle cells from injuries and death caused by oxidative stress induced by hyperglycemia.(8) In another study, rats which have their Nrf2 removed show more severe cardiac muscle injuries than those whose Nrf2 are intact after induction with streptozotocin (STZ).(9)

With the discovery of the important effect of Nrf2 in reducing oxidative stress, a DM therapy targeting this system needs to be developed. Using Nrf2 as a therapeutic target has many advantages, such as protecting cells from damage, increasing insulin sensitivity, and preventing micro and macrovascular complications. The main purpose of using Nrf2 as a therapeutic target is to adopt its ability to reduce ROS, as well as its potential to decrease peroxidation of lipid and oxidative stress in order to prevent complications.(10)

\section{Nrf2}

Nrf2 is a transcription activator which will be activated when it binds to the ARE in the promoter region of the target gene. $\mathrm{Nrf2}$ has a heterodimer formation, forming a complex with Keap1 through an interaction with leucine-zipper group. It is expressed in almost of the cells, especially in muscle tissue, including cardiac muscle, skeletal muscle, the kidneys, the lungs, and the liver. Nrf2 is located in chromosome $2 \mathrm{q} 31$. Nrf2 consists of 165,103 bases in humans, whereas in rats it ranges in size from 75.68 to $75.7 \mathrm{Mb}$.(11)

\section{Activities of Nrf2}

Normally, Nrf2 can be found binding to the Keap1 in the cytoplasm. This binding will promote the ubiquitination of
Nrf2 and its degradation by proteasome. The presence of oxidative stress will trigger a residual change of lysine in Keap1 protein, releasing Nrf2 from its bond with Keap1. Free Nrf2 will enter the nucleus to activate ARE/electrophile response element (EpRE). A signal will be formed afterward through three pathways: the mitogen-activated protein kinase (MAPK) cascade, the phosphatidylinositol 3-kinase (PI3K), and the protein kinase C (PKC).(12,13) PI3K is an important component in insulin-signalling pathways and can be activated by phenol-group antioxidants.

The movement of Nrf2 into the nucleus is also mediated by PKC. Other protein kinases, such as Protein kinase RNA-like endoplasmic reticulum kinase (PERK) and Protein kinase $\mathrm{B} / \mathrm{Akt}$, are also able to phosphorylate and activate Nrf2.(14)

\section{Roles of Nrf2}

The Nrf2 system is expressed in many cells and biological systems. Nrf2 controls several enzymes production, including antioxidative enzymes. Thus it plays an important role in cellular defense mechanisms against oxidative stress. Nrf2 is also involved in lipid metabolism and insulin action.(15) Nrf2 helps regulate insulin activity and maintain metabolic processes in body. Previous studies show that activation of Nrf2 is affected by insulinsignalling pathways. Moreover, Nrf2 activity will decrease in the presence of insulin resistance.(16)

Another study also mentions the importance of Nrf2 in improving the performance of insulin-signalling pathways, especially in the presence of insulin resistance. Circulating ROS may disrupt a normal insulin signalling pathway by causing an imbalance between reduction and oxidation, inhibiting insulin action in the process.(17) $\mathrm{Nrf} 2$ maintains the balance between reductive and oxidative reactions by reducing lipid synthesis. Thus it plays a role as an anti-obesity agent. Nrf2 activity in adipose cells inhibits adipogenesis and differentiation of immature fat cells. Maturation and differentiation of fat increases in reductionoxidation imbalances.(18)

It is hypothesized that Nrf2 reduces the differentiation of fat cells by maintaining the balance of reductive-oxidative reactions, as well as by decreasing the genes that promote adipogenesis. This mechanism is similar to the action of peroxisome proliferator-activated receptors- $\gamma$ (PPAR- $\gamma$ ).(7) Previous study shows that administration of substances which increase the activity of Nrf2 in rats fed with a high-fat diet can prevent weight gain, lower the mass of adipose tissue, and prevent a fatty liver.(19) 


\section{The ARE}

The ARE is a specific cis-acting codon formation which plays an important role in the activation mechanism of chemoprotective genes. The ARE codon formation is identified in promoter genes which are involved in chemoprotective systems, including antioxidative genes. One of the factors required in the activation of ARE is Nrf2. Activated Nrf2 entering the nucleus will bind to the ARE and form a complex with a musculoaponeurotic fibrosarcoma (Maf)G or MafK protein family which can increase ARE activity. Once activated, the ARE will increase the transcription of genes that code antioxidative genes, such as NADPH: quinone oxidoreductase-1, HO-1, and catalase.(20)

\section{Nrf2 as A Target in The Therapy of DM}

The role of Nrf2 in maintaining the homeostasis of glucose, lipid and protein metabolism, also in activity of antioxidants in the body, makes Nrf2 becomes an important target in the management of DM. Oxidative stress is known as the main cause of DM complications, making the modification of endogenic antioxidant activity, one of them through the role of Nrf2, an important objective in the therapy of DM.(21)

Nowadays, more natural substances have been proven to have the ability to activate Nrf2 in DM therapy. These natural Nrf2-activating agents include resveratrol, curcumin, catechin, and quercetin. The Nrf2-activating agents work by promoting the depolarization of membrane potential in mitochondria that will activate Nrf2. Because these agents are electrophilic, they can modify the cysteine residual complex in Keap1 protein, breaking its bond with Nrf2.(22) The Nrf2 will then enter the nucleus and avoid the degradation process. Activated Nrf2 will activate the ARE and increase the production of endogenic antioxidative enzymes. The increase of Nrf2 activity will also improve insulin sensitivity. This is what underlies the importance of $\mathrm{Nrf2}$ as a therapeutic target for DM in the future.(23)

Another advantage of using Nrf2 as a therapeutic target for DM is its role in regulating lipid and glucose metabolism. Its role in regulating lipid metabolism prevents obesity in DM patients, whereas several oral antihyperglycemic drugs reportedly cause it, especially glibenclamide. Not only that, but Nrf2 also helps protect pancreatic $\beta$-cells and increases insulin production.(24) Administration of Nrf2-activating agents in DM therapy has been widely tested in vivo, but its administration to humans still needs further research.

\section{Activation of Nrf2 by Antioxidants}

The ability of flavonoid antioxidants to increase the activity of endogenic antioxidants is related to its role in increasing the release of Nrf2 from its bond with Keap1 into the nucleus, to activate ARE/EpRE. The ARE/EpRE complex can be found in many promoter genes involved in the cellular defense mechanism. Genes such as Nrf1, Nrf2 and Nrf3 have the capacity to activate the ARE/EpRE. The Nrf 1 or 2 which binds to the ARE/EpRE will activate the genes of antioxidative enzymes which are important in regulating detoxification and the cellular defense mechanism.(22)

The Nrf1 and Nrf2 can also form a heterodimer complex with target DNA with protein basic leucine zipper domain (bZIP), MafF, and MafK. Nrf2 can also form a heterodimer complex with target DNA with proto-oncogene proteins c-Jun, activating transcription factor 4 (ATF4), polyamine-modulated factor (PMF) and PPAR- $\gamma$ which are also involved in the homeostasis of glucose and lipid.(25)

\section{Nrf2 and The Effect of Flavonoid Antioxidants on Nrf2 in Cardiac Muscles}

A population study finds that $90 \%$ of cardiomyopathies are attributed to the increase of oxidative stress in DM patients.(26) An in vitro observation of the increasing production of ROS in the presence of hyperglycemia shows an increase in apoptosis and mitochondrial injuries leading to infarction in cardiac muscles.(8) Mitochondrial injuries cause an imbalance in reductive-oxidative reactions, inhibition of electron transport in respiratory chain, and disorders in various signaling pathways.(27)

Increasing ROS and oxidative stress in cardiac muscles promote cellular apoptosis and mitochondrial injuries by mitochondrial complex II inhibitor 3-nitropropionic acid. Mitochondrial injuries will reduce the sensitivity of cardiac muscles against $\beta$-adrenergic agonist that is essential for their contraction. Not only that, mitochondrial injuries will reduce their sensitivity toward protective enzymes, including the endogenic oxidative enzymes. The Nrf2 is important in preventing infarction in cardiac muscles by decreasing ROS and oxidative stress. A study using the cardiac muscles of rats shows a higher level of ROS in those without the Nrf2. A short-term, mild hyperglycemic induction in rats without the Nrf2 causes an increase in cardiac muscle cell apoptosis, compared to in those with the Nrf2.(8) Lower expression of Nrf2 in cardiac muscles also causes nitrosative damage and 
phosphorylation of Extracellular signal-regulated kinases (ERK). Administration of agents that can increase the expression of Nrf2, such as dihydro-CDDO-trifluoromethyl amide (Dh404), can reduce nitrosative damage and phosphorylation of ERK, as well as prevent the disruption of insulin signalling pathways.(28)

Flavonoids can increase the release of Nrf2 from its bond with Keap1, allowing activated Nrf2 to enter nucleus and to activate ARE that will in turn increase the production of antioxidative enzymes. Studies shows the ability of quercetin to reduce the risk of cardiomyopathy by lowering the oxidative stress in doxorubicin-induced rats.(29)

\section{Conclusion}

Nrf2 is an essential transcription factor in the production of endogenic antioxidants. Normally, the amount of free radicals which causes oxidative stress is in the balance with the ability of endogenic antioxidants to neutralize it. In DM, however, the presence of hyperglycemia increases the amount of free radicals. Therefore, one of the main objectives in DM therapy is to increase the production and activity of endogenic antioxidants. One of the means to achieve this objective is through the administration of agents which can increase the expression and activity of Nrf2, such as flavonoid antioxidants.

\section{References}

1. Murugesh KV, Yeligar DK, Dash P, Sengupta BC, Maity TK. Antidiabetic, antioxidant and antihyperlipidemic status of Heliotropium zeylanicum extract on streptozotocin-induced diabetic rats. Biol Pharm Bull. 2006; 29: 2202-5.

2. Whiting DR, Guariguata L, Weil C, Shaw J. IDF Diabetes Atlas: Global estimates of the prevalence of diabetes for 2011 and 2030. Diabetes Res Clin Pract. 2011; 94: 311-21.

3. Riaz S. Diabetes mellitus. Sci Res Essays. 2009; 4: 367-73.

4. Perkumpulan Endokrinologi Indonesia. Konsensus Pengelolaan dan Pencegahan Diabetes Melitus Tipe 2 di Indonesia. Jakarta: PB. PERKENI; 2015.

5. Goh SY, Cooper ME. The role of advanced glycation end products in progression and complications of diabetes. J Clin Endocrinol Metab. 2008; 93:1143-52.

6. Murarka S, Movahed MR. Diabetic cardiomyopathy. J Card Fail. 2010; 16: 971-9.

7. Pi J, Zhang Q, Fu J, Woods CG, Hou Y, Corkey BE. ROS signaling, oxidative stres and Nrf2 in pancreatic beta-cell function. Toxicol Appl Pharmacol. 2010; 244:77-83.

8. He X, Kan H, Cai L, Ma Q. Nrf2 is critical in defense against high glucose-induced oxidative damage in cardiomyocytes. J Mol Cell Cardiol. 2009; 46: 47-58.
9. He X, Ma Q. Disruption of Nrf2 synergizes with high glucose to cause heightened myocardial oxidative stres and severe cardiomyopathy in diabetic mice. J Diabetes Metab. 2012; 2012 (Suppl 7): 1-9. doi: 10.4172/2155-6156.S7-002.

10. Zhi WY, Dan L, Wen HL, Tian RJ. Role of nuclear factor (erythroidderived 2)-like 2 in metabolic homeostasis and insulin action: A novel opportunity for diabetes treatment? World J Diabetes. 2012; 3: 19-28.

11. Kawai Y, Garduno L, Theodore M, Yang J, Arinze IJ. Acetylationdeacetylation of the transcription factor $\mathrm{Nrf2}$ (nuclear factor erythroid 2-related factor 2) regulates its transcriptional activity and nucleocytoplasmic localization. J. Biol. Chem. 2011; 286:7629-40.

12. Yu R, Lei W, Mandlekar S, Weber MJ, Der CJ, Wu J, et al. Role of a mitogen-activated protein kinase pathway in the induction of phase II detoxifying enzymes by chemicals. J Biol Chem. 1999; 274: 27545-52.

13. Yu R, Chen C, Mo YY, Hebbar V, Owuor ED, Tan TH, et al. Activation of mitogen-activated protein kinase pathways induces antioxidant response element-mediated gene expression via a Nrf2-dependent mechanism. J Biol Chem. 2000; 275: 39907-13.

14. Brunt KR, Fenrich KK, Kiani G, Tse MY, Pang SC, Ward CA, et al. Protection of human vascular smooth muscle cells from $\mathrm{H} 2 \mathrm{O} 2-$ induced apoptosis through functional codependence between HO-1 and AKT. Arterioscler Thromb Vasc Biol. 2006; 26: 2027-34.

15. Sykiotis GP, Bohmann D. Keap1/Nrf2 signaling regulates oxidative stres tolerance and lifespan in Drosophila. Dev Cell. 2008; 14: 7685.

16. Chang CL, Au LC, Huang SW, Kwok CF, Ho LT, Juan CC. Insulin upregulates heme oxygenase-1 expression in 3T3-L1 adipocytes via PI3-kinase- and PKC-dependent pathways and heme oxygenase-1associated microRNA downregulation. Endocrinology. 2011; 152: 384-93.

17. Bashan N, Kovsa, J, Kachko I, Ovadia H, Rudich A. Positive and negative regulation of insulin signaling by reactive oxygen and nitrogen species. Physiol Rev. 2009; 89: 27-71.

18. Ducluzeau PH, Priou M, Weitheimer M, Flamment M, Duluc L, Iacobazi F, et al. Dynamic regulation of mitochondrial network and oxidative functions during 3T3-L1 fat cell differentiation. J Physiol Biochem. 2011; 67: 285-96.

19. Shin S, Wakabayashi J, Yates MS, Wakabayashi N, Dolan PM, Aja S, et al. Role of Nrf2 in prevention of high-fat diet-induced obesity by synthetic triterpenoid CDDO-imidazolide. Eur J Pharmacol. 2009; 620: $138-44$.

20. Nerland DE. The antioxidant/electrophile response element motif. Drug Metab Rev. 2007; 39: 235-48.

21. Negi G, Kumar A, Joshi RP, Sharma SS. Oxidative stress and Nrf2 in the pathophysiology of diabetic neuropathy: old perspective with a new angle. Biochem Biophys Res Commun. 2011; 408(1): 1-5. doi: 10.1016/j.bbrc.2011.03.087.

22. Moskaug JO, Carlsen H, Myhrstad M, Blomhoff R. Molecular imaging of the biological effects of quercetin and quercetin-rich foods. Mech Ageing Dev. 2004; 125: 315-24.

23. McNally SJ, Harrison EM, Ross JA, Garden OJ, Wigmore SJ. Curcumin induces heme oxygenase 1 through generation of reactive oxygen species, p38 activation and phosphatase inhibition. Int J Mol Med. 2007; 19: 165-72.

24. Aggarwal BB. Targeting inflammation-induced obesity and metabolic diseases by curcumin and other nutraceuticals. Annu Rev Nutr. 2010; 30: 173-99.

25. Hayes JD, McMahon M. Molecular basis for the contribution of the antioxidant responsive element to cancer chemoprevention. Cancer Lett. 2001; 174: 103-13. 
26. Nishida C, Uauy, R, Kumanyika, S and Shetty P. Joint WHO/FAO Expert consultation on Diet, Nutrition and the prevention of chronic diseases: process, product and policy implications. Public Health Nutrition. 2004; 7: 245-50.

27. Duncan J G. Mitochondrial dysfunction in diabetic cardiomyopathy. Biochim Biophys Acta. 2011; 1813: 1351-9.
28. Li J, Ichikawa T, Villacorta L. Nrf2 protects against maladaptive cardiac responses to hemodynamic stres. Arterioscler Thromb Vasc Biol. 2009; 29: 1843-50.

29. Parabathina RK, Raja GV, Rao MN, Rao GS, Rao KS. Cardioprotective effects of vitamin E, morin, rutin and quercetin against Doxorubicin induced oxidative stres of rabbits: A biochemical study. J Chem Pharm Res. 2010; 2: 754-65. 